\title{
Identification of Permanent Faults for Three-phase Autoreclosing Using Inductance Parameter on Transmission Lines with Shunt Reactors
}

\author{
Zhanjun Qiao $^{1}$ and Wenbo Fan ${ }^{2}$ \\ ${ }^{1}$ Department of Mechanical and Electronic Engineering, North China Institute of \\ Science and Technology, East Yanjiao, Beijing, China \\ ${ }^{2}$ China Coal Construction Group Co,Ltd, Changping, Beijing, China \\ ${ }^{1}$ zhanjunqiao@126.com, ${ }^{2}$ fanwb@zmjsjt.com.cn
}

\begin{abstract}
A novel method using mode component inductance parameter of shunt reactors is presented in order to identify temporary faults and permanent faults for three-phase adaptive reclosure on transmission lines with shunt reactors at both ends. The method uses $\pi$-type equivalent model as the prototype to build parameter identification formula. When some single phase-to-ground fault occurs, the parameter identification formula of zero-mode component is built; when some phase-to-phase fault occurs, the parameter identification formula of line-mode component is built. The current of the shunt reactor is given and the inductance of the shunt reactor is taken as the unknown parameter to evaluate the difference between the calculated inductance and the actual one so as to distinguish permanent fault from transient fault. The inductance difference of the temporary fault is minute because the actual fault model is consistent with the prototype. But the inductance difference of the permanent fault is distinct because the actual fault model is inconsistent with the prototype. Results of EMTP simulation show that the proposed method is reliable and effective, and can be applied to the three-phase adaptive reclosure on transmission lines with shunt reactors.
\end{abstract}

Keywords: transmission line; fault identification; three-phase adaptive reclosure; shunt reactors; fault criterion

\section{Introduction}

Over the past decade, the research on identification of permanent fault is mainly directed at single-phase adaptive reclosure on transmission lines, but the research directed at three-phase adaptive is less. Compared with the traditional reclosing, three-phase adaptive reclosure technology possesses many advantages to ensure the safety and stable operation of power system, which can avoid the damage of equipment, reduce transmission capacity and even destabilize the power system.[1-3].

Generally, identification methods can be classified into three broad categories, including the method based on restore voltage in the instantaneous faults[4-6], the method based on arc characteristic in the instantaneous faults[7] and the method based on fault phase current characters of shunt reactors[8-10]. These methods have their own characteristics, and their scopes of application are also different. The method based on attenuation of high frequency signals is proposed to realize three-phase adaptive reclosure, but it is often affected by the line length, weather etc [11]. The method based on inductance parameter of shunt reactors distinguishes permanent fault from temporary fault, with $\pi$-prototype model of three-phase transmission line as reference model when some transient fault occurs, and with no decoupling between the reference models of three-phase. However, this method has complex identification formulas and large 
computation, with insufficient sensitivity when a permanent fault occurs between two phase lines [12].

A novel method using mode component inductance parameter of shunt reactors is presented in this paper, which can distinguish permanent fault from temporary fault. Using Karrenbauer transform, three-phase system can be translated into modulus system which has no coupling relationship between two phase lines, and the parameter identification formulas of zero or line-mode component can be built. The current of shunt reactor is given and the inductance of shunt reactor is taken as the unknown parameter to evaluate the difference between the calculated inductance and the actual one. Results of EMTP simulation has demonstrated the accuracy and feasibility of this method.

\section{Principle of Identifying Permanent Fault}

The sequence of three-phase reclosure can be divided into 3 stages which are shown on Figure 1, and point " 0 " is the starting moment of fault. When some fault occurs, in $0 \sim t_{2}$ period time, fault type and fault location are discriminated with relay protection device. At the moment of " $t_{2}$ ", circuit breaker trips out, and the fault is eliminated. So the permanent faults can be identified by using all kinds of data collected in $0 \sim t_{3}$ period time.

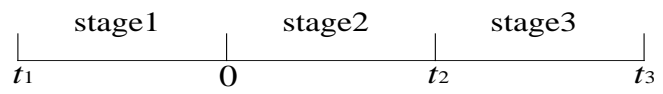

\section{Figure 1. Time Sequence of Three-phase Reclosure}

Karrenbauer transform is applied to analyze the transient process [13]. Using Karrenbauer transform, three-phase system can be translated into modulus system which contains zero-mode component and line-mode component. Based on fault type (earth faults or phase-to-phase faults) judged in $0 \sim t_{2}$ period time, the current of shunt reactor is given and the inductance of shunt reactor is taken as the unknown parameter to evaluate. Using $\pi$-type equivalent model as the prototype, some parameter identification formulas are built. When some single phase-to-ground fault occurs, the parameter identification formula of zero-mode component is built; when some phase-to-phase fault occurs, the parameter identification formula of line-mode component is built. After inductances of shunt reactor calculated, permanent faults and temporary faults can be distinguished by using the difference between the calculated inductance and the actual inductance. The inductance difference of the temporary fault is minute because the actual fault model is consistent with the prototype. But the inductance difference of the permanent fault is distinct because the actual fault model is inconsistent with the prototype.

\section{Formula of Parameter Identification for Transmission Lines with One-terminal Shunt Reactors}

\subsection{Equivalent Model for Temporary Fault of Three-phase Transmission System}

In transmission system with three-phase reclosures, when some fault occurs, three-phase circuit breaker trips out at " $t_{2}$ " moment in Figure 1, then transmission lines lose "external excitation" and become "zero-input circuit". So, the energy, which is stored in the elements of capacitances and inductances on transmission lines with shunt reactors, will continue to be discharged. When circuit breakers at both ends trip out, an oscillating circuit is formed between the capacitances of transmission lines and shunt reactors, and it presents free oscillation state.

When some temporary fault occurs, the model of three-phase transmission line with one-terminal shunt reactors is shown on Figure 2. 


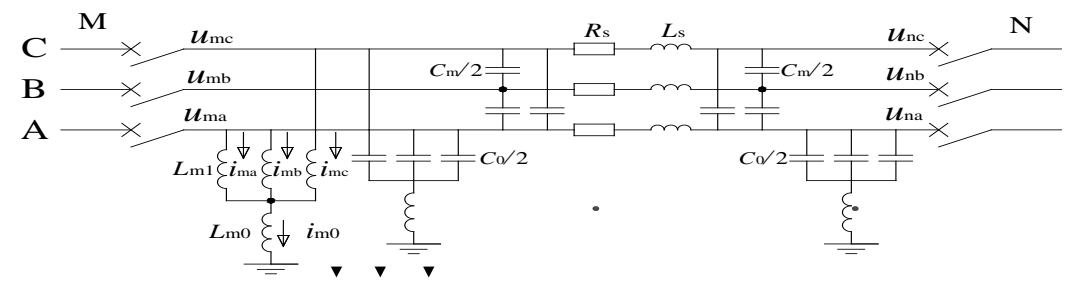

Figure 2. Equivalent Model of m-type for the Transmission Line with One-terminal Shunt Reactors

where $C_{0}$ is the shunt capacitance of transmission lines; $C_{m}$ is the capacitance between two phase lines; $L_{m 1}$ is the inductance of shunt reactors; $L_{m 0}$ is the inductance of small reactors installed on the neutral point; $L s$ is the reactance of transmission lines; $R_{s}$ is the resistance of transmission lines; $i_{m a}, i_{m b}, i_{m c}$ are current of shunt reactors for phase A, phase B and phase C at M-end respectively; $i_{m 0}$ is the current of small reactors installed on the neutral point at M-end; $u_{m a}, u_{m b}, u_{m c}$ are the phase voltage for phase A, phase $\mathrm{B}$ and phase $\mathrm{C}$ at $\mathrm{M}$-side respectively; $u_{n a}, u_{n b}, u_{n c}$ are the phase voltage for phase $\mathrm{A}$, phase $\mathrm{B}$ and phase $\mathrm{C}$ at $\mathrm{N}$-end respectively.

Normally, the influence of three-phase coupling is considered when the model of parameter identification formula is established. To simplify the parameter identification formula, some formula using zero-mode component is established based on Figure 2.

\subsection{Identification Formula based on Zero-mode Parameter}

Based on Figure 2, when some earth fault occurs, the parameter identification formula of zero-mode is established, and it is shown on Figure 3.

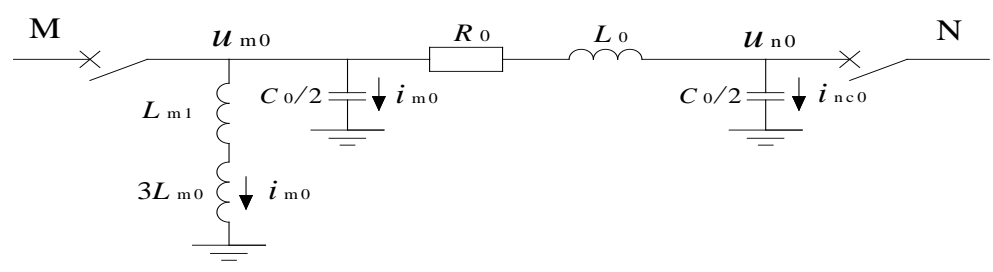

\section{Figure 3. Temporary Fault Zero Model for the Transmission Line with} One-Terminal Shunt Reactors

where $R_{0}$ is the zero-sequence resistance; $L_{0}$ is the zero-sequence reactance; $u_{m 0}$ and $u_{n 0}$ are the zero-mode voltage for $\mathrm{M}$-end and $\mathrm{N}$-end of transmission line respectively.

Based on Figure 3, zero-mode current formula is presented as:

$$
i_{m 0}+i_{m c 0}+i_{n c 0}=0
$$

where: $i_{m c 0}=\frac{C_{0}}{2} \cdot \frac{d u_{m 0}}{d t}, i_{n c 0}=\frac{C_{0}}{2} \cdot \frac{d u_{n 0}}{d t}$.

$u_{m 0}$ and $u_{n 0}$ can be calculated by the equation (2) and (3).

$$
\begin{gathered}
u_{m 0}=\left(3 L_{m 0}+L_{m 1}\right) \frac{d i_{m 0}}{d t} \\
u_{n 0}=u_{m 0}+R_{0}\left(i_{m 0}+i_{m c 0}\right)+L_{0}\left(\frac{d i_{m 0}}{d_{t}}+\frac{d i_{m c 0}}{d_{t}}\right)
\end{gathered}
$$


In order to reduce the differential order and the calculation of equation, if setting $L_{m 1}$ as the model parameter to be solved, and if substituting parameters of equation (2) and (3) into formula (1), then formula (4) as follows can be obtained.

$$
A_{0} L_{m 1}=-B_{0}
$$

where:

$$
\begin{gathered}
A_{0}=C_{0} \frac{d^{2} i_{m 0}}{d t^{2}}+k_{1} R_{0} \frac{d^{3} i_{m 0}}{d t^{3}}+k_{1} L_{0} \frac{d^{4} i_{m 0}}{d t^{4}}, \\
B_{0}=i_{m 0}+k_{2} \frac{d i_{m 0}}{d t}+k_{3} \frac{d^{2} i_{m 0}}{d t^{2}}+k_{1} k_{4} R_{0} \frac{d^{3} i_{m 0}}{d t^{3}}+k_{1} k_{4} L_{0} \frac{d^{4} i_{m 0}}{d t^{4}},
\end{gathered}
$$

and $k_{1}=\frac{C_{0}^{2}}{4}, k_{2}=\frac{R_{0} C_{0}}{2}, k_{3}=C_{0}\left(3 L_{m 0}+\frac{L_{0}}{2}\right), k_{4}=3 L_{m 0}$.

\subsection{Analysis of Temporary Fault Line Model}

Based on Figure 2, when some phase-to-phase fault occurs, the parameter identification formula of line-mode is established, and it is shown on Figure 4.

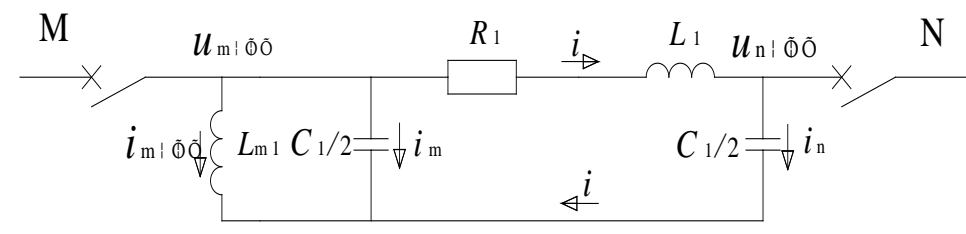

\section{Figure 4. Temporary Fault Line Model for the Transmission Line with One-terminal Shunt Reactors}

where $R_{1}$ is the positive sequence impedance; $L_{1}$ is the positive sequence reactance; $C_{1}$ is the positive sequence capacitance; $u_{m \varphi \varphi}$ and $u_{n \varphi \varphi}$ are the line-mode voltage for $\mathrm{M}$-end and $\mathrm{N}$-end of transmission line respectively.

Based on Figure 4, line-mode current formula is presented as:

$$
i_{m \varphi \varphi}+i_{m}+i_{n}=0
$$

where: $i_{m}=\frac{C_{1}}{2} \frac{d u_{m \varphi \varphi}}{d t}, i_{n}=\frac{C_{1}}{2} \frac{d u_{n \varphi \varphi}}{d t}$, and “ $\varphi \varphi$ ” represents phase line $a b, b c$, or ca respectively.

$u_{m \varphi \varphi}$ and $u_{n \varphi \varphi}$ can be calculated by the equation (6) and (7).

$$
\begin{gathered}
u_{m \varphi \varphi}=L_{m 1} \frac{d i_{m \varphi \varphi}}{d t} \\
u_{n \varphi \varphi}=u_{m \varphi \varphi}+R_{1} i+L_{1} \frac{d i}{d t}
\end{gathered}
$$

where: $i=i_{m}+i_{m \varphi \varphi}$.

Similarly, in order to reduce the differential order and the calculation of equation, if setting $L_{m 1}$ as the model parameter to be solved, and if substituting parameters of equation (6) and (7) into formula (5), then formula (8) as follows can be obtained.

$$
A_{1} L_{m 1}=-B_{1}
$$


where:

$$
\begin{gathered}
A_{1}=C_{1} \frac{d^{2} i_{m \varphi \varphi}}{d t^{2}}+k_{5} R_{1} \frac{d^{3} i_{m \varphi \varphi}}{d t^{3}}+k_{5} L_{1} \frac{d^{4} i_{m \varphi \varphi}}{d t^{4}}, \quad k_{5}=\frac{C_{1}^{2}}{4}, \\
B_{1}=i_{m \varphi \varphi}+\frac{R_{1} C_{1}}{2} \frac{d i_{m 0}}{d t}+\frac{L_{1} C_{1}}{2} \frac{d^{2} i_{m \varphi \varphi}}{d t^{2}} .
\end{gathered}
$$

And instantaneous value of $i_{m \varphi \varphi}$ at the moment of " $k$ " can be obtained with the protection device of M-end of transmission line.

\section{Formula of Parameter Identification for Transmission Lines with Two-terminal Shunt Reactors}

For the transmission lines with two-terminal shunt reactors, when some transient fault occurs, zero-mode component model and line-mode component model are shown as Figure 5.

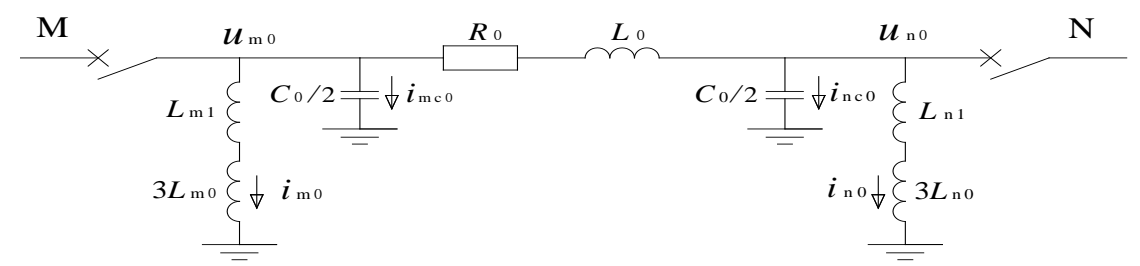

(A) Zero Mode Component Model

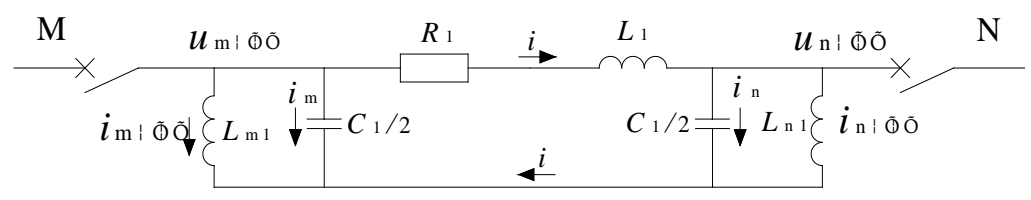

(B) Line-Mode Component Model

Figure 5. Temporary Fault Model for the Transmission Line with Two-terminal Shunt Reactors

Based on Figure 5.(a), when some earth fault occurs, the parameter identification formula of zero-mode is established and it is similar to the formula (4).

Where:

$$
A_{0}=\frac{C_{0}}{2} \frac{d^{2} i_{m 0}}{d t^{2} \mathbf{v}}, \quad B_{0}=i_{m 0}+i_{n 0}+\frac{3 C_{0} L_{m 0}}{2} \frac{d^{2} i_{m 0}}{d t^{2}}+\frac{C_{0}}{2}\left(L_{n 1}+3 L_{n 0}\right) \frac{d^{2} i_{n 0}}{d t^{2}}
$$

Similarly, based on Figure 5.(b), when some phase-to-phase fault occurs, the parameter identification formula of line-mode is established and it is similar to the formula (8).

Where:

$$
A_{1}=\frac{C_{1}}{2} \frac{d^{2} i_{m \varphi \varphi}}{d t^{2}} \quad, \quad B_{1}=i_{m \varphi \varphi}+i_{n \varphi \varphi}+\frac{C_{1} L_{n 1}}{2} \frac{d^{2} i_{n \varphi \varphi}}{d t^{2}}
$$

\section{Principle Analysis and Criterion}

\subsection{Principle Analysis}

The key idea of this method is that the inductance of shunt reactors is taken as the unknown parameter to evaluate the difference between the calculated inductance " $L_{m 1}$ " 
and the actual inductance " $L_{m 1 r}$ " so as to distinguish temporary fault from permanent fault.

In theory, when some temporary fault occurs, after circuit breakers on both ends of transmission line trip out and secondary arc extinguish, the evaluated inductance parameter is similar to actual inductance parameter and does not change with time. However, when some permanent fault occurs, the actual fault model is inconsistent with the reference model, and the difference between evaluated inductance and actual parameter is significant. So, permanent faults can be identified based on the difference between calculated inductance and actual inductance.

\subsection{The Data Processing}

Since reference model is the $\pi$-model, the high frequency components of shunt reactor current must be filtered before calculation. In this paper, a low-pass filter whose cutoff frequency is $100 \mathrm{~Hz}$ is adopted.

In formula (4) and (8), because the difference of shunt reactor current cannot be solved directly, this process can only be replaced by numerical difference approximately.

On account of the influence of various errors, when some transient fault occurs, $L_{m 1}$ always fluctuates. To improve the reliability of identification, the least squares parameter estimation is adopted in this paper. That is, the value of inductance parameter is estimated by using sampling sequence values over a period of time, and then the identification parameter sequence can be obtained with the sampling time moving forward.

\subsection{Criterion for Permanent Fault}

Setting $K(k)=\left|\sum_{i=1}^{n}\right| L_{m 1}(k+i)\left|/ n-L_{m 1 r}\right| / L_{m 1 r}$, where $L_{m 1}(k)$ is the sequence of parameters estimated by adopting the least square method, and $n=10$ is set so as to improve the identification reliability, the criterion for permanent fault is shown as:

$$
K(k)>\lambda
$$

where $\lambda$ is the margin coefficient. When some fault occurs, after circuit breakers at both ends of transmission line trip out, and if formula (9) stands repeatedly, the permanent fault can be confirmed. Otherwise the temporary fault can be confirmed.

\section{Simulation and Verification}

\subsection{The simulation system and its parameters}

Based on ATP-EMTP, some $500 \mathrm{kv}$ transmission system with one-terminal shunt reactors is proposed, and its equivalent system of double power supply is shown as Figure 6.

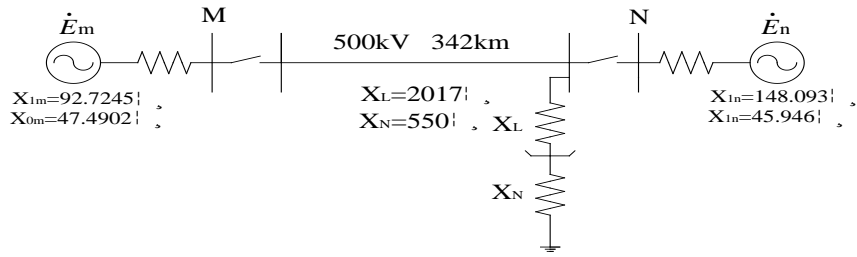

Figure 6. 500kV-transmission System with One Shunt Reactor

where: $\quad R_{1}=0.027 \Omega / \mathrm{km}, \quad R_{0}=0.1957 \Omega / \mathrm{km}, \quad L_{1}=0.9651 \quad \mathrm{mH} / \mathrm{km}$, $L_{0}=2.211 \mathrm{mH} / \mathrm{km}, C_{1}=0.0136 \mu \mathrm{F} / \mathrm{km}, \quad C_{0}=0.0092 \mu \mathrm{F} / \mathrm{km}$. 
Similarly, based on ATP-EMTP, some 500kv transmission system with two-terminal shunt reactors is proposed, and its equivalent system of double power supply is shown as Figure 7.

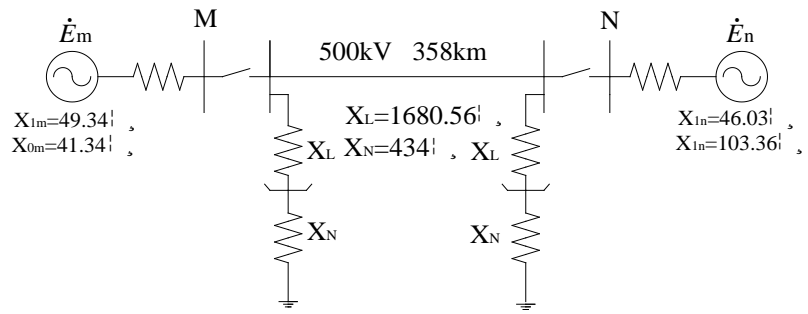

Figure 7. 500kV-transmission SYstem with Two SHUNT Reactors

where: $\quad R_{1}=0.0195 \Omega / \mathrm{km}, \quad R_{0}=0.1675 \Omega / \mathrm{km}, \quad L_{1}=0.9134 \mathrm{mH} / \mathrm{km}, \quad L_{0}=2.719 \mathrm{mH} / \mathrm{km}$, $C_{1}=0.014 \mu \mathrm{F} / \mathrm{km}, \quad C_{0}=0.00834 \mu \mathrm{F} / \mathrm{km}$.

\subsection{Simulation and Verification}

In the process of simulation, the sampling frequency is set as $2 \mathrm{kHz}$. When some transient fault occurs at the moment of $40 \mathrm{~ms}$, the circuit breaker trips out $100 \mathrm{~ms}$ later, and the secondary arc of transient fault extinguishes.

Comparing fault types, the calculated inductances of " $L_{m 1}$ " and the actual inductances of " $L_{m 1 r}$ " are shown on Figure 8 and Figure 9, where transient fault occurs at somewhere $200 \mathrm{~km}$ away from $\mathrm{N}$-end of transmission line with one-terminal shunt reactors.

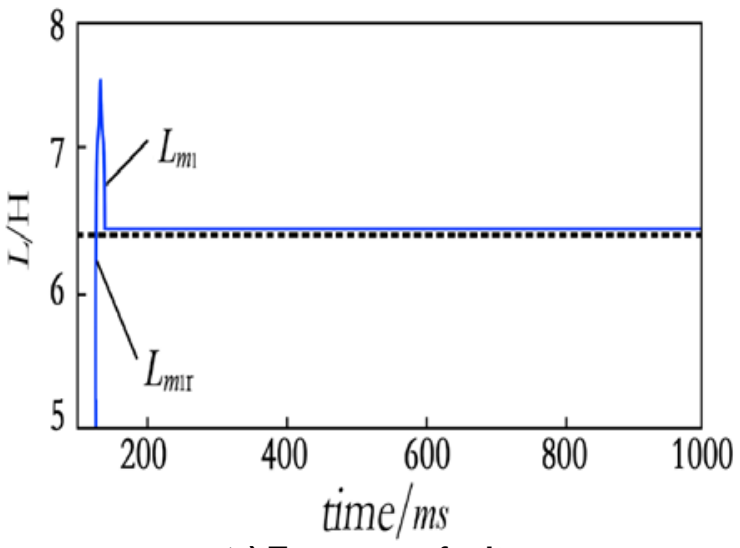

(a) Temporary fault

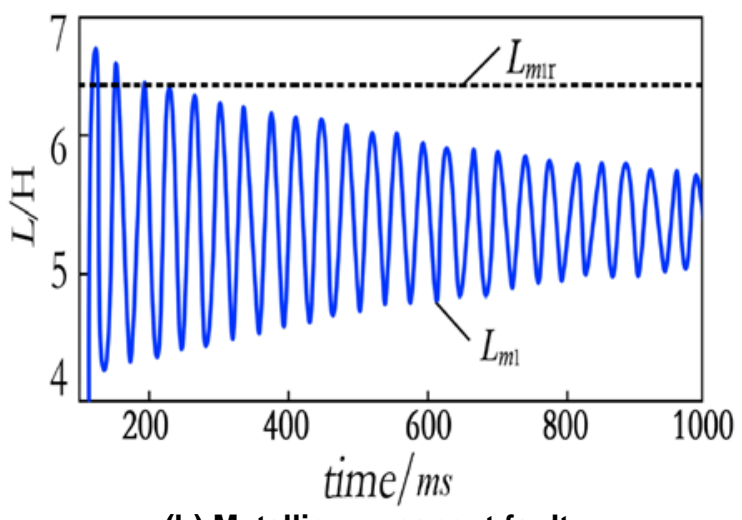

(b) Metallic permanent fault

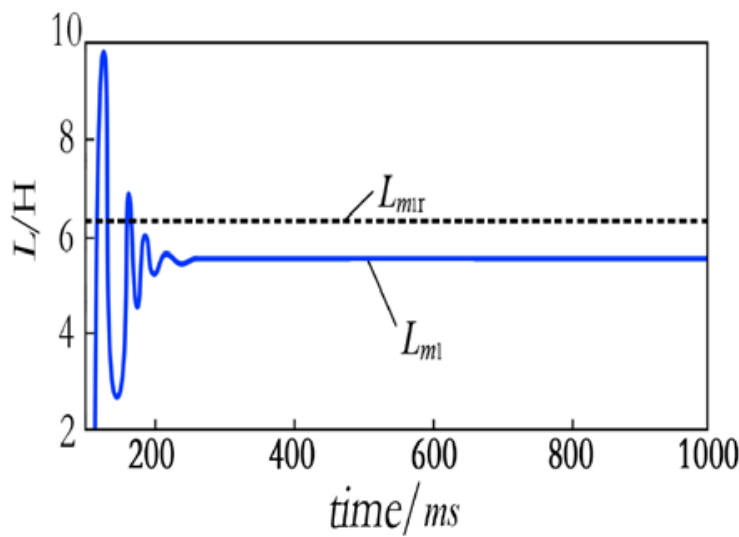

(c) Permanent fault by $300 \Omega$ transition resistance

Figure 8. Comparison for A-phase-to-ground between the Calculated Inductances and the Actual Inductances 


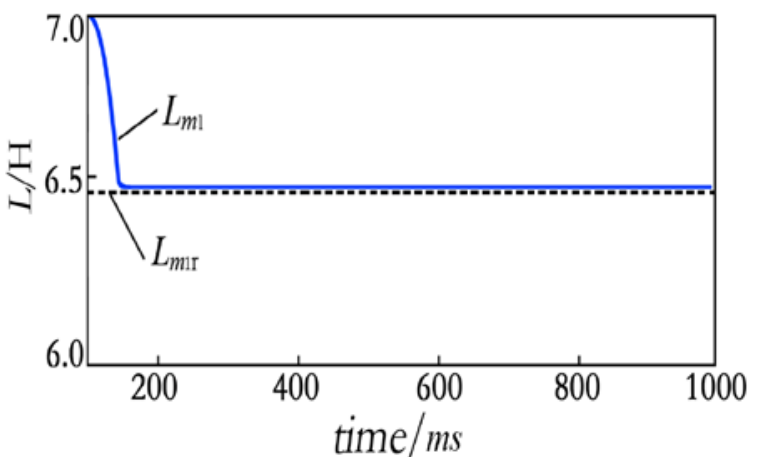

(a) Temporary fault

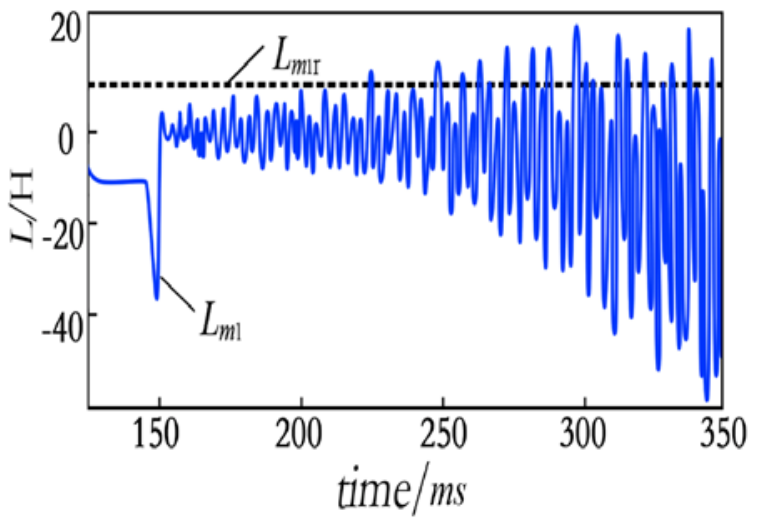

(b) Metallic permanent fault

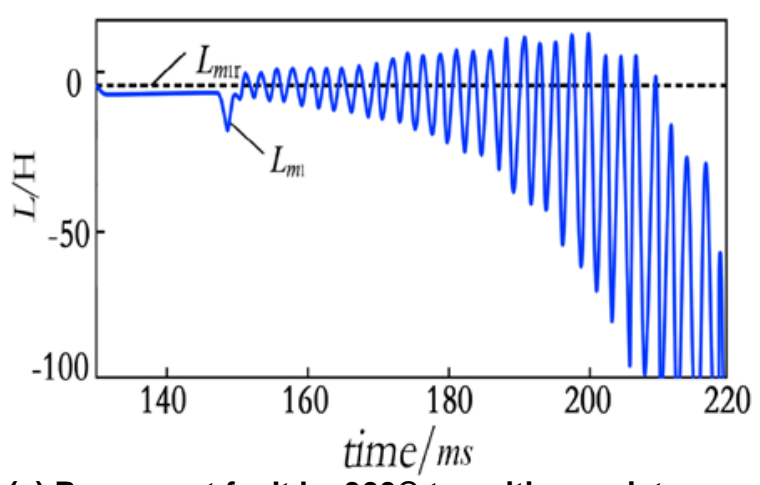

(c) Permanent fault by $300 \Omega$ transition resistance

Figure 8 and Figure 9 show: when some temporary fault occurs, after circuit breaker trips out and secondary arc extinguishes, the evaluated inductance parameter of " $L_{m 1}$ ” is similar to the actual inductance parameter of " $L_{m 1 r}$ ". However, when some permanent fault occurs, the actual fault model is inconsistent with the reference model, and " $L_{m 1}$ " deviates from " $L_{m 1 r}$ " significantly. Figure 9 (a) and (b) also show: when some phase-to-phase permanent fault occurs, energy stored in the capacitors and inductors is released quickly, and the difference between " $L_{m 1}$ " and " $L_{m 1 r}$ " increases dramatically after the circuit breaker trips out, and what's worse, short circuit by transition resistance occurs.

In order to verify the applicability of the criterion in different fault positions and transition resistance situations, simulation results are shown at the following tables corresponding to different transmission systems with shunt reactors, where the fault locations are presented by the percentage of the length of transmission line which starts from fault position to the N-end of transmission line,

and $L=\sum_{k=601}^{610}\left|L_{m 1}(k)\right| / 10, \quad K=\left|\frac{L-L_{m 1 r}}{L_{m 1 r}}\right| \times 100 \%$. “ $K$ ” presents the value of “ $K(k=165 m s)$ ” in criterion formula (9).

Table 1. Results for A-phase-to-ground with One-terminal Shunt Reactors Line System

\begin{tabular}{cccccc}
\hline \multirow{2}{*}{$\begin{array}{c}\text { Fault } \\
\text { point/\% }\end{array}$} & Resistance/ $\Omega$ & \multicolumn{2}{c}{ Temporary fault } & \multicolumn{2}{c}{ Permanent fault } \\
\cline { 2 - 6 } & & $L / H$ & $K / \%$ & $L / H$ & $K / \%$ \\
\hline \multirow{2}{*}{0} & 0.1 & 6.3792 & 0.789 & 6.8055 & 5.97 \\
\cline { 2 - 6 } & 300 & 6.4702 & 0.681 & 5.3743 & 16.31 \\
\hline \multirow{2}{*}{50} & 0.1 & 6.4697 & 0.782 & 5.9798 & 6.69 \\
\cline { 2 - 6 } & 300 & 6.3991 & 0.811 & 5.3913 & 15.89 \\
\hline \multirow{2}{*}{100} & 0.1 & 6.4701 & 0.783 & 5.6008 & 15.01 \\
\cline { 2 - 6 } & 300 & 6.4698 & 0.819 & 5.3753 & 16.47 \\
\hline
\end{tabular}


Table 2. Results for Double Line-to-ground Fault w with One-terminal Shunt Reactors Line System

\begin{tabular}{cccccc}
\hline \multirow{2}{*}{$\begin{array}{c}\text { Fault } \\
\text { point/\% }\end{array}$} & Resistance/ $\Omega$ & \multicolumn{2}{c}{ Temporary fault } & \multicolumn{2}{c}{ Permanent fault } \\
\cline { 2 - 6 } & & $L / H$ & $K / \%$ & $L / H$ & $K / \%$ \\
\hline \multirow{2}{*}{0} & 0.1 & 6.3871 & 0.966 & 7.2991 & 13.82 \\
\cline { 2 - 6 } & 300 & 6.3897 & 1.117 & 4.6908 & 25.93 \\
\hline \multirow{2}{*}{50} & 0.1 & 6.4912 & 0.824 & 4.8319 & 24.74 \\
\cline { 2 - 6 } & 300 & 6.4719 & 0.781 & 4.7103 & 26.02 \\
\hline \multirow{2}{*}{100} & 0.1 & 6.4679 & 0.763 & 4.8183 & 25.15 \\
\cline { 2 - 6 } & 300 & 6.5127 & 0.832 & 4.7079 & 25.17 \\
\hline
\end{tabular}

Table 3. Results For Line-to-line Fault with One-terminal Shunt Reactors Line System

\begin{tabular}{cccccc}
\hline \multirow{2}{*}{$\begin{array}{c}\text { Fault } \\
\text { point/\% }\end{array}$} & \multirow{2}{*}{ Resistance/ $\boldsymbol{\Omega}$} & \multicolumn{2}{c}{ Temporary fault } & \multicolumn{2}{c}{ Permanent fault } \\
\cline { 2 - 6 } & & $L / H$ & $K / \%$ & $L / H$ & $K / \%$ \\
\hline \multirow{2}{*}{0} & 0.1 & 6.3929 & 0.349 & 8792 & 128901 \\
\cline { 2 - 6 } & 50 & 6.3901 & 0.317 & 13142 & 200141 \\
\hline \multirow{2}{*}{50} & 0.1 & 6.5121 & 0.338 & 821.9 & 113972 \\
\cline { 2 - 6 } & 50 & 6.5301 & 0.319 & 6409 & 110081 \\
\hline \multirow{2}{*}{100} & 0.1 & 6.3996 & 0.321 & 0.7207 & 84.56 \\
\cline { 2 - 6 } & 50 & 6.3921 & 0.342 & 8359 & 129105 \\
\hline
\end{tabular}

Table 4. Results for Three-phase Fault with One-terminal Shunt Reactors Line System

\begin{tabular}{cccccc}
\hline \multirow{2}{*}{$\begin{array}{c}\text { Fault } \\
\text { point/\% }\end{array}$} & Resistance/ $\boldsymbol{\Omega}$ & \multicolumn{2}{c}{ Temporary fault } & \multicolumn{2}{c}{ Permanent fault } \\
\cline { 2 - 6 } & & $L / H$ & $K / \%$ & $L / H$ & $K / \%$ \\
\hline \multirow{2}{*}{0} & 0.1 & 6.3902 & 0.297 & 13917 & 201699 \\
\cline { 2 - 6 } & 50 & 6.3817 & 0.289 & 4029 & 53967 \\
\hline \multirow{2}{*}{50} & 0.1 & 6.4391 & 0.301 & 493.17 & 7627 \\
\cline { 2 - 6 } & 50 & 6.5013 & 0.321 & 849.18 & 12879 \\
\hline \multirow{2}{*}{100} & 0.1 & 6.3978 & 0.271 & 0.9760 & 81.76 \\
\cline { 2 - 6 } & 50 & 6.4629 & 0.251 & 2692.1 & 42942 \\
\hline
\end{tabular}

Table 5. Results for Line-to-line Fault with Two-terminal Shunt Reactors Line System

\begin{tabular}{cccccc}
\hline \multirow{2}{*}{$\begin{array}{c}\text { Fault } \\
\text { point/\% }\end{array}$} & Resistance/ $\Omega$ & \multicolumn{2}{c}{ Temporary fault } & \multicolumn{2}{c}{ Permanent fault } \\
\cline { 2 - 6 } & & $L / \mathrm{H}$ & $K / \%$ & $L / H$ & $K / \%$ \\
\hline \multirow{2}{*}{0} & 0.1 & 5.3713 & 1.491 & 5824 & 104927 \\
\cline { 2 - 6 } & 50 & 5.3913 & 1.523 & 8679 & 138849 \\
\hline \multirow{2}{*}{50} & 0.1 & 5.3819 & 1.536 & 116.33 & 2301 \\
\hline \multirow{2}{*}{100} & 50 & 5.6129 & 1.572 & 2405 & 42991 \\
\cline { 2 - 6 } & 0.1 & 5.5527 & 1.534 & 196.07 & 3418 \\
\hline
\end{tabular}

Table 6. Results for Three-Phase Fault with Two-terminal Shunt Reactors Line System

\begin{tabular}{cccccc}
\hline \multirow{2}{*}{$\begin{array}{c}\text { Fault } \\
\text { point/\% }\end{array}$} & Resistance/ $\Omega$ & \multicolumn{2}{c}{ Temporary fault } & \multicolumn{2}{c}{ Permanent fault } \\
\cline { 2 - 5 } & & $L / \mathrm{H}$ & $K / \%$ & $L / \mathrm{H}$ & $K / \%$ \\
\hline \multirow{2}{*}{0} & 0.1 & 5.3811 & 1.527 & 12481 & 238468 \\
\cline { 2 - 5 } & 50 & 5.2893 & 1.548 & 2478 & 42684 \\
\hline \multirow{2}{*}{50} & 0.1 & 5.2904 & 1.519 & 286.47 & 5369 \\
\cline { 2 - 5 } & 50 & 5.3921 & 1.532 & 1201 & 21698 \\
\hline \multirow{2}{*}{100} & 0.1 & 5.4287 & 1.566 & 269.89 & 5345 \\
\cline { 2 - 5 } & 50 & 5.3719 & 1.554 & 755.07 & 14014 \\
\hline
\end{tabular}

From the tables above, it is obvious that wether it is the line with shunt reactors at one end, or the line with shunt reactors at both ends, when some temporary fault occurs, the value of " $K$ " is 
smaller, and the evaluated inductance parameter is similar to actual inductance parameter. However, when a permanent fault occurs, the value of " $K$ " is larger in most cases, the actual fault model is inconsistent with the reference model, and the difference between evaluated inductance and actual parameter is significant.

\section{Conclusion}

A novel permanent fault identification method suitable for three-phase adaptive reclosure is proposed in this paper. In the presented method, the inductance parameter of mode component on transmission line with shunt reactors is used. Two parameter identification formulas are built to evaluate the difference between the calculated inductance and the actual one so as to identify temporary faults and permanent faults. In this approach, the identification formula is characterized by simplification, less calculation, high velocity, high efficiency and etc. Results of EMTP simulation show that the proposed method can reliably and effectively distinguish permanent fault from transient fault, and is suitable for the three-phase adaptive reclosure in transmission lines with shunt reactors.

\section{Acknowledgments}

We would like to acknowledge that this work was supported by "the Fundamental Research Funds for the Central Universities” (3142013065).

\section{References}

[1] W. Meiyi, “The application of power system relay protection”, Beijing: China Electric Power Press, (in Chinese), (1999), pp. 105-108.

[2] G. Yaozhong and X. Yuan, "Adaptive three phase autoreclosure of EHV transmission lines”, Electric Power Automation Equipment, (in Chinese), (1995), vol. 15, no. 2, pp. 10-18.

[3] W. Zengping, L. Haofang and X. Yan, "A new criterion for single-phase adaptive automatic reclosure based on improved correlation algorithm”, Proceedings of the CSEE, (in Chinese), vol. 27, no. 10, (2007), pp. 49-55.

[4] F. Yue and S. Wei, "Modification of voltage criterion in the single-pole automatic reclosing of transmission lines", Automation of Electric Power Systems, (in Chinese), vol. 24, no. 6, (2000), pp. 44-47.

[5] L. Bin, L. Yongli and H. Qiang, "Study on phase criterion for single-pole adaptive reclosure", Automation of Electric Power Systems, (in Chinese), vol. 27, no. 22, (2003), pp. 41-44.

[6] N. Hongzhan, D. Shuang and L. Tianyun, "Single-phase adaptive auto-reclosure based on fuzzy neural network", Power System Technology, (in Chinese), vol. 29, no. 10, (2005), pp. 75-79.

[7] M. B. Djuria and V. V. Terzija, "A new approach to the arcing faults detection for fast auto re-closure in transmission systems”, IEEE Transactions on Power Delivery, vol. 10, no. 4, (1995), pp. 793-1798.

[8] L. Bin, L. Yongli and S. Kun, "The study on single-pole adaptive reclosure of EHV transmission lines with the shunt reactor”, Proceedings of the CSEE, (in Chinese), vol. 24, no. 5, (2004), pp. 52-56.

[9] S. Wenquan, J. Lihan and S. Guobing, "Permanent fault identification method based on capacitance parameter", Journal of Xi'an Jiaotong University, (in Chinese), vol. 42, no. 6, (2008), pp. 708-712.

[10] S. Jiale, S. Dandan and F. Wei, "Identification of permanent faults for single-phase auto-reclosure on transmission lines with shunt reactors", Proceedings of the CSEE, (in Chinese), vol. 26, no. 11, (2006), pp. 75-81.

[11] L. Yongli, L. Bin and H. Qiang, "The study on three-phase adaptive reclosure based on carrier channel and signal transmission”, Proceedings of the CSEE, (in Chinese), vol. 24, no. 7, (2004), pp. 74-79.

[12] S. Wenquan, S. Guobing and S. Jiale, "Identification of permanent fault for three-phase adaptive reclosure on transmission lines with shunt reactors", Proceedings of the CSEE, (in Chinese), vol. 30, no. 4, pp. 91-98.

[13] L. Wanshun, "Power system fault analysis", $2^{\text {nd }}$ edition. Beijing: China Electric Power Press, (in Chinese), (1998), pp. 349-352.

\section{Author}

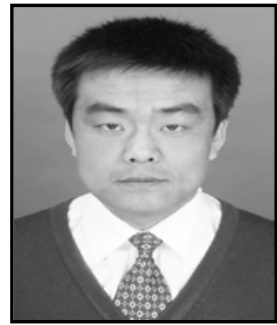

Qiao Zhanjun received PH.D. Degrees electrical engineering from the Nor th China Electric Power University (2007). Now he is an associate professor of N orth China Institute of Science And Technology. His current research interests in clude different aspects of power systems protection, fault identification and lo ad forecasting etc. 